\title{
Alterstice
}

Revue internationale de la recherche interculturelle

International Journal of Intercultural Research

Revista International de la Investigacion Intercultural

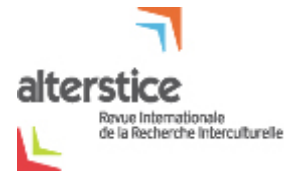

\section{Restituer la mémoire aux lieux, Prométhée au Cartucho}

\section{Michaël La Chance}

Volume 1, numéro 2, 2011

Droits humains et témoignages : l'épreuve de la culture

URI : https://id.erudit.org/iderudit/1077608ar

DOI : https://doi.org/10.7202/1077608ar

Aller au sommaire du numéro

Éditeur(s)

Alterstice

ISSN

1923-919X (numérique)

Découvrir la revue

Citer cet article

La Chance, M. (2011). Restituer la mémoire aux lieux, Prométhée au Cartucho. Alterstice, 1(2), 121-128. https://doi.org/10.7202/1077608ar

\section{Résumé de l'article}

Cet article propose une réflexion sur l'émergence du témoignage dans le cadre d'une installation urbaine par un collectif d'artistes (Mapa Teatro + C'undua de Rolf et Heidi Abderhalden). L'expérience traumatique du déplacement et la volonté politique d'effacer l'histoire d'un quartier de haute criminalité à Bogotá (Colombie) sont évoquées dans un événement théâtral, installatif et performatif : Prometeo. Cette réflexion a été proposée au colloque Arts et témoignages en contexte minoritaire, CÉLAT et LAMIC, à l’Université Laval, le samedi 2 avril 2011. 


\section{7}

alterstice

Revue Internationale

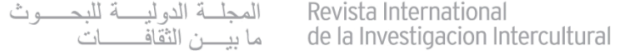

ARTICLE THÉMATIQUE

\section{Restituer la mémoire aux lieux, Prométhée au Cartucho}

Michaël La Chance ${ }^{1}$

\section{Résumé}

Cet article propose une réflexion sur l'émergence du témoignage dans le cadre d'une installation urbaine par un collectif d'artistes (Mapa Teatro + C'undua de Rolf et Heidi Abderhalden). L'expérience traumatique du déplacement et la volonté politique d'effacer l'histoire d'un quartier de haute criminalité à Bogotá (Colombie) sont évoquées dans un événement théâtral, installatif et performatif : Prometeo. Cette réflexion a été proposée au colloque Arts et témoignages en contexte minoritaire, CÉLAT et LAMIC, à l'Université Laval, le samedi 2 avril 2011.

\section{Rattachement de l'auteur}

${ }^{1}$ Département des arts et lettres, Université du Québec à Chicoutimi, Saguenay, Canada

\section{Correspondance}

michael_lachance@uqac.ca

\section{Mots clés}

intervention urbaine; performance; Colombie

\section{Pour citer cet article :}

La Chance, M. (2011). Restituer la mémoire aux lieux, Prométhée au Cartucho. Alterstice, 1(2), 121-128. 


\section{Introduction}

On voit les fils de ces vies se dérouler, s'enrouler, se mêler, et chercher à se nouer au destin de Prométhée. Lulle (2004)

Nous voulons examiner un projet d'art engagé qui fait appel aux témoignages de personnes minorisées, quand ces témoignages sont validés par une réciprocité de paroles et d'écoutes. En effet, un récit de vie est validé par le simple fait qu'il suscite d'autres récits, chacun disant: moi aussi j'ai une histoire à raconter. Ainsi, un certain nombre de personnes qui avaient été expulsées d'un quartier à haut risque de Bogotá, en Colombie, sont revenues sur les lieux, chacun n'ayant rien à gagner de témoigner sinon de participer à un événement commémoratif à caractère théâtral - sinon d'appeler d'autres témoignages qui contribuent cumulativement à reconstituer une mémoire collective. L'analyse de ces témoignages filmés nous fournit également l'occasion de livrer une réflexion personnelle sur la valeur de témoignage de la création artistique, à une époque où le poète et l'artiste sont relégués dans une minorité créatrice.

\section{El Cartucho et le rocher de Prométhée}

Dans un terrain vague de Bogotá, entre les quartiers Santa Inés et San Bernardo, à proximité de la rue El Cartucho, s'est joué un événement théâtral. II a été qualifié tour à tour d'installation théâtrale, d'événement urbain, d'art en situation urbaine, "instalacción o acto performativa(o) ». Le metteur en scène Rolf Abderhalden parle d' «instalacción ». En fait ces appellations manquent une dimension essentielle : ce sont des œuvres réalisées à partir du vécu de personnes et l'œuvre, c'est la rencontre de ces vécus.

El Cartucho était un quartier très appauvri et dégradé du centre de Bogotá, caractérisé par la prostitution et le crime, le trafic de drogues et d'armes, la pauvreté extrême d'une forte population de sans-abri, d'enfants et de toxicomanes. Ce quartier de vingt mille mendiants, assassins, voleurs, drogués et membres de gangs compte une forte proportion de déplacés par la guerre civile. Ce sont des paysans et aussi des minorités autochtones, qui se retrouvent soudain entassés les uns sur les autres dans des immeubles abandonnés, des crack-houses ou sur les trottoirs. Privations de toute sorte, absence d'hygiène, hébétude de vivre au jour le jour dans la misère, perversions engendrées par la souffrance et le déplacement, taux d'addiction le plus important au monde, etc. : la violence est omniprésente au Cartucho, la police n'ose pas entrer dans ce que le documentariste Stan Guigui appelle un « magma de sang, de crasse et de violence » (Guigui, 2010).

Au fil des ans, El Cartucho est devenu une excroissance monstrueuse au centre de Bogotá (7 millions d'habitants), un État dans l'État. Situation étonnante : les minorités occupent le centre, devenu un des endroits les plus dangereux de la Colombie. C'est pourquoi, malgré sa valeur historique et patrimoniale, ce quartier a été rasé par les pouvoirs municipaux en 1998. Des milliers d'habitants ont été déplacés, certains pour la deuxième fois, des centaines de parcelles démolies, pour que naisse ce qui est aujourd'hui le Parc Tercer Milenio, bien plat et ouvert à la vue, avec des allées et du gazon - mais qui reste un endroit assez dangereux : je me suis promené autour en autobus, je n'ai pas osé en descendre.

L'événement Prometeo, monté par Mapa Teatro - Laboratorio de Artistas, sur deux années (2002-2003), est un appel au témoignage. Les expulsés du Cartucho ont été invités à parler : pour se réapproprier un lieu dont ils ont été dépossédés de façon abrupte dans une opération de purification urbaine, lorsque les bulldozers sont arrivés en même temps que la police, et pour redonner une signification à un lieu qui a été plongé dans l'amnésie (Mapa Teatro, 2003).

Même si les immeubles ont été rasés, les individus ont conservé la mémoire de leurs emplacements et de leurs habitants, et plusieurs regrettent leur vieux quartier malgré la criminalité et la misère qui s'étaient progressivement installées dans les années 1970. C'était l'enfer au El Cartucho, et pourtant de nombreuses personnes ont regretté d'en sortir : on y faisait de vraies rencontres, des relations profondes et durables se tissaient à travers l'entraide et les deuils. Ces expulsés, qui ont été dispersés sans amélioration de leurs conditions de vie, diront alors qu'au Cartucho ils étaient de vraies personnes, le réel était plus réel. Curieusement il semble que cette cristallisation de la 
réalité dans le quartier Santa Inés serait corrélative de l'acquisition d'une valeur mythologique: ce lieu et cette époque étaient bien réels, car en cette époque l'humanité du Cartucho était un Prométhée de douleur prisonnier d'un rocher. Le quartier rasé devient un rocher déserté par l'agonie divine, un lieu de mémoire, un espace pour accueillir la parole.

Ainsi, dans le cadre du projet Prometeo, d'anciens résidents du quartier sont revenus sur les lieux, pour mêler leurs témoignages sur la vie au Cartucho à des lectures de passages choisis d'un grand récit mythologique: le Prométhée de Heiner Müller, qui repose sur le grand texte d’Eschyle. Ai-je mentionné que ces témoignages et lectures ont été filmés et projetés en temps réel sur un écran géant? Et que ces comédiens assortissaient leurs lectures d'un commentaire sur le texte de Heiner Müller qu'ils avaient en main ?

Cet événement public a proposé une parole où s'enchevêtraient trois discours : les récits de vie, le grand récit (de H. Müller) et les commentaires sur le grand récit. Comme si l'intégration des témoignages personnels dans un discours mythologique donnait à ceux-ci une efficacité performative: les immeubles réapparaissent. C'est une réappropriation des lieux, pour la durée de l'événement, lorsque les expulsés réinstallent quelques pièces de mobilier (chambre à coucher, salon, toilette) à l'emplacement précis de leur maison rasée. Le terrain vague devient un musée éphémère d'objets familiers, les expulsés évoluent autour de ce mobilier comme au temps jadis, ils portent les vêtements qui commémorent les grands moments de leur vie d'antan au Cartucho. Peut-être leurs premiers jours dans les bas-fonds, peut-être leur mariage, peut-être les obsèques d'un proche : ils deviennent les acteurs de leur mémoire, laquelle est consolidée de se nouer à d'autres mémoires. Le témoignage est vivant, nous pouvons de nouveau vivre les événements de notre passé, nous serons de nouveau transformés par ceux-ci.

À cet enchevêtrement de paroles entre le personnel et le mythique s'ajoutait un enchevêtrement des régimes de l'image : les récits de vie théâtralisés et projetés en direct sur l'écran composaient avec des extraits d'archives filmiques, dont certains remontaient à 1950, et où on voyait les tramways circuler dans le quartier Santa Inés, les façades bourgeoises et l'agitation intense dans le paysage urbain.

Une quinzaine d'anciens habitants ont répondu à l'appel du groupe d'artistes C'undua. Avec leurs vêtements de circonstance, quelques pièces de mobilier posées à même le sol et leurs récits édifiants, ils ont servi d'amorce à l'événement. Ces premiers joueurs sont représentatifs de la population d'El Cartucho, diverse par ses minorités. Ce premier contingent est formé d'une prostituée et de quelques mariachis, un unijambiste, une femme Noire, des Autochtones chassés de la forêt par la violence politique, etc. Tout au long de l'événement-témoignage, ce petit groupe d'acteurs improvisés sera visité par des membres de leurs familles et d'anciens voisins, des nostalgiques et des curieux, tous des survivants d'une époque. II a été possible de vérifier sur place le niveau de participation, et de prêter attention au rapport de force entre artistes et résidents. L'artiste, disposant d'équipements électroniques sophistiqués de traitement du son et de l'image, reconstitue la figure de l'écrivain public, prêt à recueillir les doléances d'une minorité traumatisée. Cependant, il faut se méfier des effets démagogiques de la spectalisation de l'expérience privée : la réécriture du témoignage afin de produire des images projetées plus grandes que nature. En fait, l'authenticité du témoignage n'est pas attestée par le caractère traumatique de l'expulsion ou de la violence de la vie passée dans le Cartucho, mais par la validation partagée.

Les anciens résidents nous ont proposé leur interprétation personnelle du mythe de Prométhée, ils ont fait connaître d'emblée les aspects qui les avaient touchés. Nous voulons en signaler quelques-uns : la souffrance infinie, le bas-fond excrémentiel, l'exploitation sans limite et aussi la normalisation urbaine. Dans les quelques pages qui suivent, nous mettrons en relief ces quatre aspects du témoignage des minorités déplacées et expulsées, avant d'aborder un propos concomitant : l'art en tant que témoignage.

La plupart diront que Prométhée dévoré par l'aigle est l'image de la souffrance infinie. En effet, selon Robert Graves :

Zeus fit enchaîner Prométhée, nu, à une colonne dans les montagnes du Caucase où un vorace vautour lui dévorait le foie toute la journée, du début à la fin de l'année. Et il n'y avait pas de terme à sa souffrance, car toutes les nuits (au cours desquelles Prométhée souffrait cruellement du froid et du gel), son foie se reconstituait. (Graves, 1989, t.I, p. 159) 
Il est vrai que les habitants du Cartucho peuvent aisément s'identifier à la figure du Prométhée sacrifié, enchaîné à son rocher - pour peu que le Prométhée grec s'apparente au Señor Caido de Monserrate. Un Christ de souffrance dont le sarcophage de verre est exhibé au sommet (3152 m) de la cordillère orientale. C'est un pèlerinage annuel de tous les habitants, mais en certaines occasions la statue du seigneur déchu est descendue au centre-ville de Bogotá pour les malades, les vieillards, les nécessiteux. Le Prométhée christique est arraché à son rocher pour être exhibé aux hommes, bouleverser leur ferveur, leur rappeler l'infini de la souffrance. Le Christ, disait Nietzsche dans la Généalogie de la morale, a été sacrifié une fois pour toutes, et connaîtra une sublimation finale (La Chance, 1995, p. 72). Tandis que Prométhée exhibe une souffrance sans fin.

Le Cartucho offre l'image d'une souille fondamentale de l'humanité, le bas-fond d'une agressivité anale, quand - selon Heiner Müller - «l'aigle, qui le prenait pour un morceau de roche partiellement comestible, capable de faire des petits mouvements et d'émettre un chant dissonant, surtout quand il le mangeait faisait aussi ses besoins sur lui. Cet excrément était son aliment. II le rendait, transformé en son excrément, sur la pierre d'en bas. " (Müller, 1982). Nous sommes dans un tel cloaque, avec un étiolement des valeurs humaines : nous ne pouvons pas donner plus que nous avons reçu. Qui a reçu de la souffrance, infligera de la souffrance. Qui a reçu de I'amour donnera de l'amour. Il y a une économie des affects à la base de nos existences, une entropie de sentiments qui conduit vers une plus grande abjection. Le Cartucho illustre une dernière dégradation de l'humanité dans son autodestruction tragique.

D'autres voudront voir dans l'aigle noir une figure du capitalisme dévorant, un emblème de la mondialisation qui accapare les ressources. Un homme du Cartucho dit de l'aigle qu'il « dévorait tous les jours l'argent de mes poches que je devais remplir sans m'arrêter » (cité par Lulle, 2004). Prométhée c'est le peuple qui ne cesse d'être exploité, un peuple qui a peur de sa liberté. Après qu'Héraclès eut abattu l'aigle, Prométhée s'accroche à son rocher, il se voue au supplice. Le Prométhée de Müller

défendit ses chaînes avec dents et ongles, contre l'intervention du libérateur. Une fois délivré, marchant sur les mains et les genoux, hurlant du supplice d'avoir à se mouvoir avec des membres engourdis, il réclamait à grands cris la place tranquille qu'il avait sur la pierre, sous l'aile protectrice de l'aigle. (Müller, 1982).

Prometeo c'est l'inertie d'une population qui ne peut quitter sa condition de victime, lorsque les minorités se découvrent incapables de retrouver leurs responsabilités d'acteurs sociaux, et se croient incapables d'une créativité culturelle.

Quatrième aspect du témoignage et des commentaires des minorités expulsées : la normalisation urbaine. En fait, la démolition du quartier El Cartucho, qui était fort dégradé spatialement et socialement, coïncide avec un mouvement mondial de redéfinition de l'espace social. Avec la fragmentation urbaine, l'atomisation des ensembles sociaux, les pouvoirs publics effacent toute trace de désobéissance civile. La ville de Bogotá aspire à devenir une métropole de verre et d'acier qui reste parfaitement étanche aux contaminations par les idéologies de subversion (rebelles des FARC) ou par les animismes des paysans et des Autochtones. Les pouvoirs publics ont entrepris de reconquérir le centre, ils se sont ensuite empressés de tout raser, afin d'empêcher tout retour possible des populations. D’où la nécessité d'un centre vide, un terrain-vague-gazonné au cœur de la métropole, lequel devient un moteur de déshumanisation : "La métropole est la mort simultanée de la ville et de la campagne. " (Le comité invisible, 2002, p. 40).

La métropolisation de Bogotá a transformé El Cartucho en un terrain nivelé qui a reçu l'appellation faussement historique de parc Tercio Milenio. Il fallait en reconstituer l'histoire, ce qui a été rendu possible lorsque, dans un climat de confiance, les témoignages ont commencé à affluer. Cette mise en commun des souffrances occasionnées par l'exode rural et la misère urbaine, puis par l'expulsion, a permis à la parole d'acquérir un statut politique. Mieux encore, la dimension de la rencontre s'est révélée éminemment politique, même si cette rencontre n'a pas été utilisée pour ventiler des revendications contre la municipalité et contre le gouvernement. Mapa Teatro a réalisé une œuvre intitulée Testigo de las ruinas, à mi-chemin entre la vidéo-installation et le théâtre documentaire, qui reprend l'essentiel des interventions, dramaturgies et témoignages (Mapa Teatro, 2007). 


\section{Prométhée et la minorité créatrice}

Après avoir présenté certains aspects du témoignage des personnes minorisées, dans l'événement Prometeo, je voudrais proposer un questionnement sur notre capacité à recueillir ces témoignages - proposer un témoignage sur le témoignage qui mette en relief une autre minorité, la minorité créatrice des artistes et des poètes. Je crois qu'il est utile de témoigner en faveur de cette minorité lorsque nous voulons interroger le rôle joué par les artistes dans l'appel au témoignage des anciens du Cartucho. Ce rôle pourrait apparaître suspect lorsqu'il s'agit de régénérer la représentation à partir du trauma, sinon de réinvestir le sens à partir du sang. Un processus que nous avons observé à maintes reprises : la capitalisation de la souffrance pour produire du spectacle (La Chance, 2007).

En fait, lors de mon passage au Mapa Teatro à Bogotá, quand j’ai pris connaissance des documents vidéos entourant le projet Prometeo, j'ai été particulièrement touché par le drame humain du Cartucho, et aussi par l'effort de ses habitants d'interroger leur vie en regard d'un mythe, quand " on voit les fils de ces vies se dérouler, s'enrouler, se mêler, et chercher à se nouer au destin de Prométhée " (Lulle, 2004). Le regroupement d'artistes C'undua sera parvenu à transposer la doléance individuelle en un moment poétique qui appartient en propre à la condition humaine. Elle acquiert une force et une noblesse qui lui fait côtoyer les mythes. Les témoignages se réclament de Prométhée, mais ils s'enchevêtrent aussi entre eux. Ici, les acteurs/résidents sont venus témoigner dans le contexte d'une sacralisation de la mémoire : mon destin noué à celui de Prométhée. On se demande quelle est la valeur de l'intervention artistique : est-ce que l'art témoigne pour l'humanité? Quel est le rapport entre la poésie et l'impasse de la condition humaine?

Prométhée devient le visage de l'humanité sacrifiée, c'est aussi une image de l'art et de la poésie en général. J'ai été touché par l'interprétation du mythe par les déplacés du Cartucho, et je me suis aussi senti personnellement interpellé par cette invocation. J'ai reconnu le travail de l'artiste qui vient donner sa dernière représentation dans un paysage post-apocalyptique. Lors d'une soirée de reconstitution-témoignage, un ancien résident est revenu avec son costume de clown, pour commémorer ses années de saltimbanque. Je me suis reconnu dans cette figure dérisoire et grimée, son inadéquation tragique, sa fierté lorsqu'il exhibe sa boîte à surprises, dans un dernier numéro de nuit, sur un terrain marqué par les chenilles des bulldozers.

Il fut un temps où le poète était façonneur de monde - de l'Antiquité au Romantisme, en passant par la Renaissance. La société tout entière est un processus autopoïétique, elle se façonne comme œuvre d'art, elle est noyau de gestation dans lequel l'homme de civilisation se donne naissance à lui-même. Mais, Hegel l'aura déclaré haut et fort, l'art est mort, c'est-à-dire que la poésie comme l'art ont perdu cette puissance fondationnelle. Alors, prétendre jouer ce rôle aujourd'hui relève effectivement, de la part du poète et de l'artiste, de la clownerie. Tel le clown qui nous offre un moment de cirque lorsque, chancelant sur les ruines du monde, il arrache une fleur de sa blessure. Il peut encore espérer nous offrir une " expérience privée », au sens d’Agamben :

Les puissances historiques traditionnelles - poésie, religion, philosophie - qui, tant dans la perspective hégélo-kojévienne que dans celle de Heidegger, tenaient en éveil le destin historico-politique des peuples, ont été depuis longtemps transformées en spectacles culturels et en expériences privées, et ont perdu toute efficacité historique. (Agamben, 2002, p. 124)

Et pourtant, le clown de El Cartucho a offert son témoignage, figure de l'innocence sacrifiée, du vieillard perpétuellement enfant, et qui perdure. Qu'en est-il de l'art qui recueille les témoignages en contexte traumatique ? Quand l'art lui-même est une forme de témoignage dans le contexte des droits bafoués ? Comment cette voix peut-elle se faire entendre à l'ère d'une uniformisation culturelle qui fait la promotion de l'anonymat et du tout-fonctionnel?

Dans un monde subjugué par une vaste narcose culturelle, l'artiste et le poète font véritablement partie d'une minorité créatrice. Selon Ortega y Gasset :

I'homme-masse croit effectivement qu'il est l'État, et qu'il tendra de plus en plus à le faire fonctionner sous n'importe quel prétexte, pour anéantir grâce à lui toute minorité créatrice qui le gêne - qui le gêne dans n'importe quel domaine : dans celui de la politique, de l'industrie, aussi bien que dans celui des idées. (Ortega y Gasset, 2011, p. 196) 
J'ai la conviction que les œuvres stimulantes opèrent un transfert de créativité dans le public. J'ai aussi une crainte : que cette créativité soit reléguée à une minorité d'individualistes et de désespérés, ou du moins refusant les faux espoirs de la science, des promesses électorales et de la religion. C'est ainsi que les artistes et les écrivains sont confinés loin du centre : on les a relégué dans un isolement, celui de la minorité créatrice, où ils disqualifient leur propre parole. Le poète écrit un livre pour briser son isolement, qui sera lu par d'autres poètes qui écriront des livres pour témoigner de leur isolement. Ainsi de l'art : parce qu'ils sont convaincus de la valeur mythique de l'art et de la poésie, l'artiste et le poète érigent leur quotidien à la hauteur d'un mythe et tout en se faisant dévorer par ce mythe. Le poète et l'écrivain n'échappent pas au supplice de la réécriture, l'artiste s'impose la nécessité de repartir à zéro. Comme s'ils avaient trouvé dans le mythe de la littérature et de l'art les instruments et le protocole de leur propre torture. André Gide avait attiré l'attention sur cet aspect du mythe de Prométhée : l'aigle, c'est les passions qui s'alimentent de la substance de l'homme. C'est l'artiste qui cannibalise sa vie au nom de l'art, qui sacrifie son quotidien pour affirmer une réalité des œuvres et qui, éventuellement, trouvera une réalité à sa vie, à son quotidien, pourvu qu'il bénéficie d'une réelle reconnaissance dans l'expression de l'humain.

Avec l'événement Prometeo, l'exercice initial a consisté à mêler notre voix à une parole hiératique qui parle de dévoration et d'excrément, de sacrifice et de mort libératrice. Chacun a entrepris de se nouer au mythe, là où nous voulons éternellement dénouer la souffrance. Je voudrais moi aussi poser une chaise sur la terre battue du chantier, et voir les fils de ma vie, selon la belle expression de Thierry Lulle, "se dérouler, s'enrouler, se mêler, et chercher à se nouer au destin de Prométhée ».

Quand chacun, pour peu qu'il soit porteur d'une œuvre, peut s'écrier: en quoi suis-je moi aussi Prométhée? Epiméthée est "celui qui pense après coup ". Prométhée : celui qui pense auparavant, qui aura voulu penser le destin de l'humanité, qui aura donné de sa personne pour le penser. C'est la transgression de Prométhée, avoir vu avant l'heure tous les maux de l'humanité. Et, parce qu'il anticipe cette souffrance, parce qu'il participe déjà à celle-ci, il voit la nécessité d'enfermer tous les maux dans une jarre - la boîte dite de Pandore, car c'est elle Pandore, la belle-sœur de Prométhée, qui l'ouvrira par après. La transgression de Prométhée, c'est d'avoir rêvé d'un monde sans folie. Robert Graves appuie son récit sur Hésiode et le Scholiaste d'Apollonios de Rhodes :

[Pandore] ouvrit une jarre, que Prométhée avait recommandé à son frère [Épiméthée] de tenir close et dans laquelle il avait eu le plus grand mal à enfermer tous les maux capables d'affliger le genre humain : notamment la vieillesse, le travail, la maladie, la folie, le vice et la passion. Tous les maux se répandirent au-dehors en une immense nuée et piquèrent Epiméthée et Pandore sur toutes les parties de leurs corps puis s'attaquèrent aux mortels. (Graves, 1989, t. I, p. 159)

Depuis le commentaire inaugural d'Aristote sur le poème dramatique, le poète et l'artiste sont invités à nous révéler que nous avons une boîte de Pandore en nous: des blessures et des souffrances que nous acceptons de regarder à condition de se leurrer qu'il s'agit du désespoir d'un autre, qu'il s'agit de paroxysmes qui ne nous appartiennent pas. À condition d'avoir l'assurance que nous saurons aussitôt refermer la boîte. Nous aurons entrevu le chaos, assurés d'une clôture de la représentation. Voilà pourquoi nous restons prisonniers de notre rocher, car nous avons accepté l'idée que l'humanité ne saura plus sortir de l'impasse. Que nous ne saurons en aucun cas accéder à un monde meilleur.

Lorsque l'humanité a le ventre déchiré, il ne sert à rien de recueillir des témoignages pour en faire des œuvres d'art. II y a cependant dans le projet Prometeo une interrogation fondamentale sur le devenir de l'humain, il ne s'agit pas tant d'extraire ces témoignages de personnes minorisées pour en tirer des effets de vérité, il ne s'agit pas tant de faire des récits de vie une revendication de justice, il s'agit plutôt de poser une question : comment rester sains d'esprit devant la souffrance des déplacés, des sidéens, de sans-abri qui ne sauraient témoigner? Le dispositif artistique nous conduit à cette interrogation: comment sommes-nous parvenus à recueillir les témoignages de gens qui ont perdu la raison tant ils ne savent réagir aux demandes de leur environnement que par la violence et l'intoxication? Formulé autrement : à quoi tient notre santé mentale lorsqu'elle nous permet d'accepter un monde d'inégalités et d'injustices, lorsque nous devenons spectateurs d'une humanité réduite au statut de bête blessée? Je parle ici du quartier El Cartucho comme tel. 
II y a, en effet, un lien essentiel qui relie le témoignage personnel et l'espérance du vivre ensemble. Le vivre ensemble présuppose une qualité d'espoir, une santé de la condition humaine qui n'est pas l'espoir trompeur que crée une utopie politique ou religieuse. La version que donne Robert Graves du mythe de Prométhée est accablante sur ce point : "Cependant la trompeuse Espérance, que Prométhée avait aussi enfermée dans la jarre, les dissuada, par ses mensonges, d'un suicide général. " (Graves, 1989). Prométhée prend la pleine (dé)mesure de l'impasse du genre humain, du problème humain pour lequel il n'y a pas de solution, sinon l'autodestruction. II nous laisse avec la question du rôle de l'art et de la poésie devant l'impasse.

La réponse religieuse traduit un esprit qui a cédé devant l'absurdité du mal, qui ne peut accepter que tant de souffrance soit inutile, qui finit par établir une économie primaire qui relie la vérité et la douleur. Le témoignage du trauma dans le dispositif artistique est-il exempt de cette économie? L'infinie souffrance du Señor Caido au sommet de la cordillère est la figure sacrificielle d'une souffrance millénaire : c'est le Prométhée de Bogotá. Quelle réponse non religieuse pouvons-nous apporter quand la création artistique (théâtre, littérature, etc.) pourrait témoigner en faveur de l'humain, ses valeurs d'entraide et de cohabitation? En fait, nous ne saurions recueillir ces témoignages, si nous les écoutions vraiment. Et tout à la fois ils doivent être lus et entendus parce que c'est ainsi que nous forgeons une qualité d'espoir.

\section{Ce que dit en d'autres termes Flannery O'Connor,}

Les gens désespérés n'écrivent pas de romans, pas plus qu'ils n'en lisent. Ils ne portent pas un regard soutenu sur les choses, ils n'en ont pas le courage. Le refus de se mettre à l'épreuve conduit au désespoir, tandis que le roman, bien sûr, conduit à l'expérience. (Flannery O'Connor, 1969)

La littérature permet ce regard soutenu, quand une qualité d'espoir permet un engagement auprès de nos contemporains - certes, Beckett, Bernhard, Cioran et d'autres démentent cette approche. L'art nous permet de croire que la reconnaissance culturelle est fondatrice d'une communauté éthique. Sinon, nous n'avons de nouvelles du monde que dans une litanie de guerres et de catastrophes, dans des récits mis en forme par nos médias et nos gouvernements. Qu'est-ce que je connais du monde qui m'entoure? Je suis spectateur des fictions articulées par nos oligarchies, édulcorées par le devoir de neutralité de nos médias. Quelle est l'ampleur du pillage de la nature? Comment puis-je me connecter aux enjeux humains de cette époque, quand tout ce que je connais du monde est un tableau de pixels? Mes voyages récents, tel ce voyage en Colombie dont je parle maintenant, et aussi Cuba, le Brésil, m'ont montré que ce qu'on voit sur place ne correspond en rien à ce qu'on peut en dire. Et les témoignages que je peux entendre, lorsqu'il s'agit de corruption, d'enlèvement et de tortures, de l'exploitation des enfants, etc., dans les quartiers de misère, me font prendre conscience que je ne pouvais pas imaginer le degré d'abjection auquel l'être humain peut descendre.

Alors comment croire à la reconnaissance réciproque et interculturelle lorsqu'il semble que nous n'aurions que l'avancée technologique pour nous sauver ici-bas, et la religion pour nous sauvegarder dans l'au-delà. Comment croire à une décence minimale de l'être humain, à une aspiration naturelle vers une meilleure forme de vie, à la transcendance intérieure dont parle Axel Honneth (2000)? Comment puis-je espérer de véritables transformations, sur les plans économiques et culturels, de ma société? Nous ne pouvons plus rien attendre? Sommes-nous une génération perdue? Sommes-nous parvenus à une nouvelle frontière? Ou plutôt, sommes-nous au pied du mur? Pour ma part, j'interroge la pertinence de ce que je fais, qui consiste essentiellement à écrire et enseigner; je m’interroge sur l'impact réel de la poésie et des arts, lorsqu'il s'agit de créer une identification émotionnelle à l'autre, et de créer une reconnaissance intersubjective. Dans le bas-fond du Cartucho, documenté par Stan Guigui, il n'y a plus de minorités, sinon une sous-humanité à laquelle nous sommes devenus indifférents. Le projet Prometeo fait réapparaître les particularismes, fait revivre une diversité où l'individu est réinstitué comme expérience subjective et point de vue.

Prometeo, monté par Mapa Teatro en plusieurs actes, m'apparaît comme une illustration forte du rôle de l'art dans l'élaboration du vivre ensemble. Il ne suffit pas de dissiper l'illusion généralisée d'un progrès de nos conditions de vie en société. II ne suffit pas non plus de reconnaître l'ampleur de la crise de l'humanité. II faut créer de réelles situations de reconnaissance interculturelles et intersubjectives. Il me semble que pour ma part je pratique une écriture-refuge, que mes textes sont des collections d'artefacts émotionnels, que mes œuvres sont des matrices 
pour absents. Cela m'inquiète, et tout à la fois je me réjouis de constater que ma démarche n'est pas si différente d'un résident du Cartucho auquel on a confié le Prométhée de Heiner Müller. Je peux le comprendre sans me placer moi aussi, l'ouvrage de Müller à la main, parmi les ruines.

\section{Remerciements}

Je veux remercier Rolf et Heidi Abderhalden (Mapa Teatro et C'undua) et Ximena Vargas (Mapa Teatro) pour la documentation photographique et vidéo. Et aussi, pour m’avoir fait connaître le Mapa Teatro à Bogotá : Ricardo Arcos-Palma (Chaire francocolombienne d'histoire de l'architecture et de l'art, Bogotá) et Constanza Camelo (UQAC).

\section{Références bibliographiques}

Agamben, G. (2002). L'Ouvert. De l'homme et de l'animal (J. Gayrand, trad.). Paris : Rivages [coll. Petite Bibliothèque].

Graves, R. (1989). Les mythes grecs (trad.). Paris : Hachette [coll. Pluriel].

Guigui, S. (producteur) (2010). El Cartucho, "Le royaume des voleurs » [documentaire]. Cape Town (Afrique du Sud) : Pistoleros Films.

Honneth, A. (2000). La lutte pour la reconnaissance. Paris : Cerf.

La Chance, M. (1995). Artaud et Nietzsche : une métaphysique des forces. Dans S. Harel (dir.), Antonin Artaud, figures et portraits vertigineux (p. 63-72). Montréal : XYZ éditeur.

La Chance, M. (2007). CEuvres-bombes et bioterreur. Québec : Inter / Marseille : Nouvel Al Dante.

Le comité invisible. (2002). L'insurrection qui vient. Paris : La Fabrique éditions.

Lulle, T. (2004). Prométhée contre la fragmentation urbaine. Multitudes, 17, 175-182.

Mapa Teatro (producteur) (2003). Prometeo Primera Parte [Vidéo, 19 min]. En ligne sur le site : http://vimeo.com/20983464 [Mot de passe : mapacundua2002].

Vargas, X. (productrice) (2007). Testigo de las ruinas [Vidéo, 1h 14]. En ligne à : http://vimeo.com/19431640

Müller, H. (1982). La mission. Suivi de : Prométhée. Vie de Gundling.... Quartett. Paris : Les Éditions de Minuit.

O'Connor, F. (1969). The Nature and Aim of Fiction. Dans S. et R. Fitzgerald (dir.), Mystery and Manners: Occasional Prose (p. 63-86). New York : Farrar Straus Giroux.

Ortega y Gasset, J. (2011). La révolte des masses. Paris : Les Belles Lettres. 